\title{
Susceptibilidad a antimicrobianos en aislamientos de Streptococcus pneumoniae invasor en Colombia
}

\author{
Aura Lucía Leal, ${ }^{1}$ Elizabeth Castañeda ${ }^{1}$ y \\ Grupo Colombiano de Trabajo en Streptococcus pneumoniae ${ }^{2}$
}

RESUMEN Se realizó un estudio para determinar los patrones de susceptibilidad a los antimicrobianos de los aislamientos de Streptococcus pneumoniae causante de enfermedad invasora diagnosticada en Colombia en niños menores de 5 años entre 1994 y 1996 y para establecer la distribución de los tipos capsulares de los aislamientos resistentes. Se analizaron 324 aislamientos recuperados durante la ejecución del Protocolo Nacional de Serotipificación de S. pneumoniae realizado en Santa Fe de Bogotá, Medellín y Cali, Colombia, entre julio de 1994 y marzo de 1996. Se observó que 119 de todos los aislamientos (36,7\%) presentaban susceptibilidad disminuida por lo menos a un antimicrobiano, que 39 (12\%) presentaban susceptibilidad disminuida a la penicilina y que de estos últimos aislamientos, 29 presentaban resistencia intermedia y 10 resistencia alta. Nueve aislamientos $(2,8 \%)$ presentaban resistencia a la ceftriaxona, $80(24,7 \%)$ a la combinación de trimetoprima y sulfametoxazol (TMS), 49 (15,1\%) al cloranfenicol y $31(9,6 \%)$ a la eritromicina. Se observó resistencia a dos antimicrobianos en 31 aislamientos (9,6\%) y multirresistencia en $22(6,7 \%)$. Estos 22 aislamientos mostraron resistencia al TMS. Las asociaciones más frecuentes fueron penicilina, TMS y eritromicina en 5 casos; penicilina, cloranfenicol, TMS y eritromicina en 4; penicilina, ceftriaxona, cloranfenicol y TMS en 3; y penicilina, ceftriaxona, cloranfenicol, TMS y eritromicina en 3 casos. Los serotipos más frecuentes en los aislamientos resistentes a la penicilina fueron: $23 \mathrm{~F}(53,8 \%), 14(25,6 \%), 6 \mathrm{~B}$ $(7,7 \%), 9 \mathrm{~V}(5,1 \%), 19 \mathrm{~F}(5,1 \%)$ y $34(2,6 \%)$. Los serotipos más frecuentes en los aislamientos resistentes a antimicrobianos distintos de la penicilina fueron: $5(37,5 \%), 23 F(7,5 \%), 14$ $(18,8 \%)$ y $6 B(13,8 \%)$. Esta diferencia en la distribución de los serotipos fue estadísticamente significativa $(\mathrm{P}<0,0001)$. Los resultados de este estudio indican la necesidad de mantener una vigilancia activa de los patrones de susceptibilidad a los antibióticos para evitar la resistencia de S. pneumoniae y de suministrar información oportuna para modificar los hábitos de prescripción y consumo de antimicrobianos.

La aparición y diseminación de aislamientos de Streptococcus pneumoniae con resistencia a la penicilina y a otros

\footnotetext{
1 Instituto Nacional de Salud, Grupo de Microbiología, Santa Fe de Bogotá, Colombia. La correspondencia debe dirigirse a E. Castañeda a la siguiente dirección postal: Avenida El Dorado, Carrera 50, Zona 6, Santa Fe de Bogotá, Colombia.

2 La lista de participantes aparece en el anexo 1.
}

agentes antimicrobianos provocan un serio problema de salud pública en varias regiones del mundo (1). La situación es particularmente delicada si se tiene en cuenta que S. pneumoniae continúa siendo el principal agente etiológico de la neumonía bacteriana y el segundo de la meningoencefalitis y que, además, se asocia en ambos casos con una mortalidad alta (2). Aunque desde su introducción la penicilina ha sido el agente antimicrobiano de elección para tratar la infección neumocócica, desde el primer informe de resistencia a la penicilina en 1967 y especialmente en las dos últimas décadas, países como España, Sudáfrica y Hungría han dado a conocer tasas altas de resistencia a ese fármaco (3-5). S. pneumoniae también ha presentado resistencia a algu- 
nos otros antibióticos utilizados para combatirlo, entre ellos el cloranfenicol, la eritromicina, la combinación de trimetoprima y sulfametoxazol (TMS) y, recientemente, las cefalosporinas de tercera generación $(6,7)$. Se ha demostrado una clara asociación entre la resistencia de $S$. pneumoniae a la penicilina y a las cefalosporinas de tercera generación y una evolución clínica desfavorable en pacientes con meningitis (8). En consecuencia, es indispensable conocer los patrones de resistencia a esos antimicrobianos en cada país (9) para poder establecer las pautas terapéuticas adecuadas.

Si bien esa información existe en muchas partes del mundo, la situación no era muy conocida en la mayoría de los países de América Latina $(10,11)$. En 1993, la Organización Panamericana de la Salud (OPS) diseñó un protocolo para establecer los tipos capsulares de $S$. pneumoniae que causan enfermedades invasoras en niños menores de 5 años. La iniciativa se ejecutó en el marco del Sistema Regional de Vacunas (SIREVA) con la financiación conjunta de la Agencia Canadiense para el Desarrollo Internacional (CIDA) (12). El estudio correspondiente se realizó simultáneamente en Colombia y en otros cinco países de América Latina (Argentina, Brasil, Chile, México y Uruguay). Los criterios de inclusión comprendieron la presencia de cualquier enfermedad invasora causada por S. pneumoniae en los aislamientos de sangre y de líquido cefalorraquídeo o cualquier líquido normalmente estéril. Se excluyeron los cuadros clínicos con una evolución de más de 15 días y los casos de estridor laríngeo o crup. En Colombia, el tamaño de la muestra se calculó según el número de aislamientos necesarios para establecer la prevalencia de los tipos capsulares en el país: se necesitaron 203 aislamientos para encontrar una prevalencia de tipos capsulares de $5 \%$, con una precisión absoluta de $2 \%$ y un intervalo de confianza de $95 \%$ (13).

El objetivo del presente estudio fue determinar los patrones de susceptibilidad a antimicrobianos de los aislamientos de S. pneumoniae obtenidos durante el estudio citado y establecer la distribución de los tipos capsulares de los aislamientos resistentes. El estudio se llevó a cabo de julio de 1994 a marzo de 1996 como parte del Protocolo Nacional de Serotipificación de S. pneumoniae que se efectuó en Colombia (13).

\section{MATERIALES Y MÉTODOS}

\section{Aislamientos}

Se estudiaron 324 aislamientos de S. pneumoniae recuperados de niños menores de 5 años con infección invasora que fueron atendidos en los principales centros pediátricos de Santa Fe de Bogotá, Medellín y Cali, Colombia, entre 1994 y 1996. Los cuadros clínicos correspondieron a 133 neumonías, 132 meningitis, 36 sepsis y 21 infecciones de otros tipos; y no se obtuvo el diagnóstico de 2 pacientes. No se excluyó a los pacientes que habían recibido antibióticos. Todos los aislamientos fueron serotipificados utilizando el panel de antisueros del Statens Seruminstitut de Dinamarca (14).

\section{Susceptibilidad a antimicrobianos}

Todos los aislamientos fueron sometidos a la prueba tamiz para la penicilina por el método de difusión en disco (Kirby Bauer) mediante el empleo de discos de oxacilina de $1 \mu \mathrm{g}$ (15). Se determinó la concentración inhibidora mínima (CIM) de la penicilina, la ceftriaxona y el TMS con el método de microdilución en caldo Mueller Hinton, ajustado con cationes (DIFCO) y con el agregado de sangre lisada de caballo al 5\% (16). La CIM a la eritromicina y al cloranfenicol se estableció con la prueba Épsilon (E-test) (17). Para determinar la susceptibilidad a la vancomicina se utilizó la prueba de difusión en disco (15). En todos los casos se siguieron los parámetros establecidos por el Comité Nacional Regulador de Estándares del Laboratorio Clínico (National Committee for Clinical Laboratory Standards-NCCLS) de los Estados Unidos de América $(15,16)$. Se utilizó como cepa de control S. pneu- moniae ATCC 49619 y se definió la multirresistencia como resistencia a tres o más familias de antimicrobianos.

\section{Análisis estadístico}

Se realizó un análisis univariado de los datos describiendo las frecuencias individuales. Para el análisis bivariado se calculó la razón de prevalencias (RP), definida como una medida de asociación análoga al riesgo relativo que se aplica en estudios transversales, y se usaron intervalos de confianza de 95\%. Para determinar la significación estadística de la comparación de proporciones se usaron la prueba de ji al cuadrado $\left(\chi^{2}\right)$ y la de Fisher. El análisis estadístico se realizó con el programa Epi Info (versión 6.03) $(18,19)$.

\section{RESULTADOS}

De los 324 aislamientos estudiados, $205(63,3 \%)$ fueron sensibles a todos los antimicrobianos probados y 119 $(36,7 \%)$ presentaron susceptibilidad disminuida a uno o más antimicrobianos (cuadro 1). La prueba tamiz con oxacilina detectó 39 (12\%) aislamientos con sensibilidad disminuida a la penicilina (SDP). De estos, 29 (8,9\%) presentaron resistencia intermedia (CIM $0,1-1,0 \mu \mathrm{g} / \mathrm{mL})$ y $10(3,1 \%)$ fueron altamente resistentes (CIM $\geq 2,00 \mu \mathrm{g} / \mathrm{mL}$ ). De los aislamientos con resistencia intermedia a ese antimicrobiano, 21 (72,4\%) presentaron una CIM que osciló entre 0,125 y $0,250 \mu \mathrm{g} / \mathrm{mL}$, y en 8 $(80 \%)$ de los aislamientos altamente resistentes la CIM fue de $2 \mu \mathrm{g} / \mathrm{mL}$. Los porcentajes de resistencia se mantuvieron constantes durante todo el estudio. Nueve aislamientos $(2,8 \%)$ presentaron susceptibilidad reducida a la ceftriaxona, 8 con resistencia intermedia (CIM de $1 \mu \mathrm{g} / \mathrm{mL}$ ) y solo un aislamiento fue altamente resistente (CIM = $2 \mu \mathrm{g} / \mathrm{mL})$. Se encontró resistencia al cloranfenicol en 49 casos (15\%) y la CIM osciló entre 8 y $48 \mu \mathrm{g} / \mathrm{mL}$. Ochenta aislamientos $(24,7 \%)$ presentaron resistencia al TMS; de los 46 aislamientos con resistencia intermedia, $33(72 \%)$ presentaron una CIM de $1 \mu \mathrm{g} / \mathrm{mL}$ y 23 
CUADRO 1. Susceptibilidad a los antibióticos en 324 aislamientos de Streptococcus pneumoniae. Colombia, 1994-1996

\begin{tabular}{|c|c|c|c|c|c|c|c|c|}
\hline \multirow[b]{3}{*}{ Antibiótico } & \multicolumn{8}{|c|}{ Susceptibilidad a los antibióticos } \\
\hline & \multicolumn{2}{|c|}{ Sensibles } & \multicolumn{2}{|c|}{$\begin{array}{l}\text { Con resistencia } \\
\text { intermedia }\end{array}$} & \multicolumn{2}{|c|}{$\begin{array}{c}\text { Con resistencia } \\
\text { alta }\end{array}$} & \multicolumn{2}{|c|}{ Total } \\
\hline & No. & $\%$ & No. & $\%$ & No. & $\%$ & No. & $\%$ \\
\hline Penicilina & 285 & 88,0 & 29 & 8,9 & 10 & 3,1 & 39 & 12,0 \\
\hline Ceftriaxona & 315 & 97,2 & 8 & 2,5 & 1 & 0,3 & 9 & 2,8 \\
\hline Cloranfenicol & 275 & 85,0 & 0 & - & 49 & 15,0 & 49 & 15,0 \\
\hline TMS $^{\mathrm{a}}$ & 244 & 75,3 & 46 & 14,2 & 34 & 10,5 & 80 & 24,7 \\
\hline Eritromicina & 293 & 90,4 & 24 & 7,4 & 7 & 2,2 & 31 & 9,6 \\
\hline Vancomicina & 324 & 100,0 & 0 & - & 0 & - & 0 & - \\
\hline
\end{tabular}

a TMS = Trimetoprima y sulfametoxazol.

$(68 \%)$ de los 34 aislamientos altamente resistentes presentaron una CIM de $\geq 8 \mu \mathrm{g} / \mathrm{mL}$. Se determinó que 31 aislamientos $(9,6 \%)$ tenían sensibilidad disminuida a la eritromicina. Todos los aislamientos $(n=324)$ fueron sensibles a la vancomicina (cuadro 1 ).

Se establecieron 18 patrones de resistencia (cuadro 2); 66 (20,4\%) aislamientos presentaron resistencia a un solo antimicrobiano, $31(9,6 \%)$ a dos, y

CUADRO 2. Patrones de resistencia de 119 aislamientos de Streptococcus pneumoniae con sensibilidad disminuida a los antimicrobianos. Colombia, 1994-1996

\begin{tabular}{lrr}
\hline \multicolumn{1}{c}{ Antibiótico $^{\text {a }}$} & No. & $\%$ \\
\hline P & 8 & 2,5 \\
C & 16 & 4,9 \\
TMS & 33 & 10,2 \\
E & 9 & 2,8 \\
P, C & 1 & 0,3 \\
P, TMS & 8 & 2,5 \\
P, E & 1 & 0,3 \\
C, TMS & 14 & 4,3 \\
TMS ,E & 3 & 0,9 \\
C, E & 4 & 1,2 \\
P, C, TMS & 3 & 0,9 \\
P, CEF, TMS & 2 & 0,6 \\
P, TMS ,E & 5 & 1,5 \\
C, TMS, E & 1 & 0,3 \\
P, CEF, C, TMS & 3 & 0,9 \\
P, C, TMS, E & 4 & 1,2 \\
P, CEF, TMS, E & 1 & 0,3 \\
P, CEF, C, TMS, E & 3 & 0,9 \\
Total & 119 & 36,7 \\
\hline
\end{tabular}

$\mathrm{P}=$ penicilina; $\mathrm{C}=$ cloranfenicol; $\mathrm{TMS}$ = trimetoprima más sulfametoxazol; $\mathrm{E}=$ eritromicina; $\mathrm{CEF}=$ ceftriaxona
$22(6,7 \%)$ a tres o más. Las asociaciones más frecuentes en casos de resistencia a dos fármacos fueron cloranfenicol y TMS en 14 casos (4,3\%), penicilina $\mathrm{y}$ TMS en $8(2,5 \%)$ y cloranfenicol y eritromicina en 4 casos (1,2\%). Entre los aislamientos multirresistentes, las asociaciones más frecuentes fueron penicilina,TMS y eritromicina en 5 casos $(1,5 \%)$; penicilina, cloranfenicol, TMS y eritromicina en 4 casos $(1,2 \%)$; penicilina, ceftriaxona, cloranfenicol y TMS en 3 casos $(0,9 \%)$ y penicilina, ceftriaxona, cloranfenicol, TMS y eritromicina en 3 casos $(0,9 \%)$.

La resistencia a otros antimicrobianos fue significativamente mayor en el grupo de aislamientos con SDP. Todos los aislamientos con resistencia marcada a la penicilina (10/10) y $72,4 \%$ $(21 / 29)$ de los que tenían resistencia intermedia presentaron susceptibilidad reducida a otros antimicrobianos; por lo tanto, la prevalencia de resistencia a otro antibiótico fue de 2,58 en los aislamientos con resistencia intermedia a la penicilina (RP: 2,58; IC95\%: 1,93 a 3,45) y aumentó a 3,56 cuando el aislamiento presentaba alta resistencia (RP: 3,56; IC95\%: 2,96 a 4,99; $\chi^{2}=42,78$; $P<0,0001)$.

Veintiuno de los 22 aislamientos multirresistentes mostraron resistencia a la penicilina. La prevalencia de multirresistencia fue 38 veces mayor en los aislamientos con SDP $(48,7 \%)$ que en los aislamientos con susceptibilidad disminuida a antimicrobianos distin- tos de la penicilina (1,25\%) (RP: 38,97; IC95\%: 6,41 a 280,63). Todos los aislamientos multirresistentes mostraron resistencia a TMS.

En el grupo de los aislamientos con SDP, el mayor porcentaje de resistencia correspondió a TMS $(74,4 \%)$, seguido por el cloranfenicol $(35,9 \%)$, la eritromicina $(23,1 \%)$ y la ceftriaxona $(23,1 \%)$. Todos los aislamientos con resistencia a la ceftriaxona fueron resistentes a la penicilina. Se encontró una asociación entre la resistencia a TMS y SDP (RP: 4,16; IC95\%: 3,05 a 5,66). La asociación con SDP se presentó también en los casos resistentes al cloranfenicol (RP: 2,92; IC95\%: 1,73 a 4,93 ) y a la eritromicina (RP: 6,02; IC95\%: 3,23 a 11,22).

\section{Serotipos}

La resistencia a antimicrobianos se distribuyó en 12 de los 34 tipos capsulares determinados en el estudio de referencia (13). De los aislamientos con algún patrón de resistencia, $81 \%$ pertenecieron a los serotipos 5, 6B, 14 y 23F. Los tipos capsulares de los 39 aislamientos con SDP fueron $23 \mathrm{~F}(53,8 \%)$, $14(25,6 \%), 6 \mathrm{~B}(7,7 \%), 9 \mathrm{~V}(5,1 \%), 19 \mathrm{~F}$ $(5,1 \%)$ y $34(2,6 \%)$. Entre los 80 aislamientos con susceptibilidad disminuida a antimicrobianos distintos de la penicilina, los serotipos más frecuentes fueron $5(37,5 \%), 23 \mathrm{~F}(7,5 \%), 14(18,5 \%)$ y $6 \mathrm{~B}(13,8 \%)$. Esta diferencia en la distribución de los serotipos fue estadísticamente significativa $(P<0,0001)$ (cuadro 3). De los 31 aislamientos del serotipo 23F, $21(67,8 \%)$ presentaron resistencia a la penicilina (RP: 12,83; IC95\%: 7,85 a $20,97)$. De manera similar, 30 de los 34 $(88,2 \%)$ aislamientos del serotipo 5 fueron resistentes al cloranfenicol (RP: 12,37; IC95\%: 7,92 a 19,31).

Se encontró un mayor porcentaje de aislamientos resistentes en los niños menores de 2 años (95/238, 40\%) en comparación con los mayores de esa edad $(24 / 86,28 \%)$, pero esta diferencia no fue estadísticamente significativa (RP: 1,43; IC95\%: 0,98 a 2,08). Tampoco se encontraron diferencias significativas en la distribución de la resistencia según sexo, diagnóstico clínico o fuente de los aislamientos. 
CUADRO 3. Distribución de los tipos capsulares de 119 aislamientos de Streptococcus pneumoniae con susceptibilidad disminuida a los antimicrobianos. Colombia, 1994-1996

\begin{tabular}{|c|c|c|c|c|c|c|c|}
\hline \multirow[b]{3}{*}{ Serotipo } & \multirow{3}{*}{$\begin{array}{c}\text { Aislamientos } \\
\text { No. }\end{array}$} & \multicolumn{6}{|c|}{ Aislamientos con susceptibilidad reducida a los antimicrobianos } \\
\hline & & \multicolumn{2}{|c|}{$\begin{array}{l}\text { Con resistencia } \\
\text { a la penicilina }{ }^{a} \\
\qquad(n=39)\end{array}$} & \multicolumn{2}{|c|}{$\begin{array}{l}\text { Sin resistencia } \\
\text { a la penicilina }{ }^{\mathrm{b}} \\
\qquad(n=80)\end{array}$} & \multicolumn{2}{|c|}{$\begin{array}{c}\text { Total } \\
(n=119)\end{array}$} \\
\hline & & No. & $\%$ & No. & $\%$ & No. & $\%$ \\
\hline 1 & 29 & 0 & - & 2 & 6,9 & 2 & 6,9 \\
\hline 5 & 34 & 0 & - & 30 & 88,2 & 30 & 88,2 \\
\hline $6 \mathrm{~A}$ & 20 & 0 & - & 3 & 15,0 & 3 & 15,0 \\
\hline $6 \mathrm{~B}$ & 29 & 3 & 10,3 & 11 & 37,9 & 14 & 48,3 \\
\hline $9 \mathrm{~V}$ & 4 & 2 & 50,0 & 1 & 25,0 & 3 & 75,0 \\
\hline 14 & 71 & 10 & 14,1 & 15 & 21,1 & 25 & 35,2 \\
\hline $18 \mathrm{C}$ & 16 & 0 & - & 1 & 6,3 & 1 & 6,3 \\
\hline $19 B$ & 3 & 0 & - & 1 & 33,3 & 1 & 33,3 \\
\hline $19 \mathrm{~F}$ & 23 & 2 & 8,7 & 5 & 21,7 & 7 & 30,4 \\
\hline $23 \mathrm{~F}$ & 31 & 21 & 67,8 & 6 & 19,4 & 27 & 87,1 \\
\hline $28 \mathrm{~A}$ & 3 & 0 & - & 3 & 100,0 & 3 & 100,0 \\
\hline 34 & 3 & 1 & 33,3 & 2 & 66,7 & 3 & 100,0 \\
\hline
\end{tabular}

\section{Distribución geográfica}

Los porcentajes y patrones de resistencia mostraron algunas variaciones geográficas (cuadro 4). La resistencia a la penicilina, a la ceftriaxona y a TMS se concentró en Santa Fe de Bogotá, donde $14 \%$ de los 150 aislamientos recuperados presentaron SDP, en contraste con $12,4 \%$ de los 89 aislamientos de Medellín y 8,9\% de los 67 aislamientos de Cali. Sin embargo, esta diferencia no fue estadísticamente significativa. Al comparar la proporción de aislamientos con alta resistencia a la penicilina obtenidos en Santa $\mathrm{Fe}$ de Bogotá $(9 / 150,6 \%)$ y en las otras ciudades $(1 / 174,0,5 \%)$, se encontró una diferencia estadísticamente significativa $\left(\chi^{2}=6,22 ; P=0,01\right)$. De la misma manera, la diferencia fue significativa cuando se comparó la resistencia a la ceftriaxona $(P=0,014)$ y a TMS $(P=$ $0,003)$ en Santa Fe de Bogotá y en las otras ciudades. Los aislamientos resistentes al cloranfenicol fueron $19,8 \%$ en Cali, $18 \%$ en Medellín y $12 \%$ en Santa Fe de Bogotá, pero la diferencia no fue estadísticamente significativa. Aunque Medellín presentó el mayor porcentaje de multirresistencia $(10,1 \%)$ en comparación con Santa Fe de Bogotá $(8,7 \%)$ y Cali $(3 \%)$, esta diferencia tampoco fue significativa $(P=0,38)$.

\section{DISCUSIÓN}

El conocimiento de los patrones de susceptibilidad a los antimicrobianos en un área geográfica determinada es de vital importancia para determinar las pautas de manejo clínico de la infección neumocócica. Los resultados de este estudio muestran que $63,3 \%$ de los aislamientos obtenidos de pacientes con infecciones invasoras eran susceptibles a la penicilina o a algún otro antimicrobiano. Debe notarse que el porcentaje de los aislamientos con SDP $(12 \%)$, aunque inferior al registrado en España (40\%) y Hungría (56\%), supera los valores de otras regiones de Europa (20) y se asemeja al de Pakistán (21). Si bien los datos publicados en Colombia sobre la resistencia de S. pneumoniae a los antimicrobianos son escasos y se refieren a aislamientos nasofaríngeos de niños con neumonía (SDP de 17\%) (22), dichos datos son similares a los encontrados en este estudio. Además, la experiencia de otros países también indica la necesidad de mantener la vigilancia para detectar aumentos o cambios en los patrones de resistencia (23).

La detección de aislamientos altamente resistentes a la penicilina en 3\% de los casos es relevante porque los

CUADRO 4. Distribución por ciudades de los 324 aislamientos de Streptococcus pneumoniae y la susceptibilidad a los antimicrobianos. Colombia, 1994-1996

\begin{tabular}{|c|c|c|c|c|c|c|c|c|c|c|c|}
\hline \multirow[b]{3}{*}{ Ciudad } & \multirow{3}{*}{$\begin{array}{c}\text { Total de } \\
\text { aislamientos } \\
\text { No. }\end{array}$} & \multicolumn{10}{|c|}{ Susceptibilidad reducida a los antimicrobianos } \\
\hline & & \multicolumn{2}{|c|}{ Penicilina } & \multicolumn{2}{|c|}{ Ceftriaxona } & \multicolumn{2}{|c|}{ Cloranfenicol } & \multicolumn{2}{|c|}{$\mathrm{TMS}^{\mathrm{a}}$} & \multicolumn{2}{|c|}{ Eritromicina } \\
\hline & & No. & $\%$ & No. & $\%$ & No. & $\%$ & No. & $\%$ & No. & $\%$ \\
\hline Bogotá & 150 & 21 & 14,0 & 8 & 5,3 & 18 & 12,0 & 49 & 32,7 & 11 & 7,3 \\
\hline Medellín & 89 & 11 & 12,4 & 1 & 1,1 & 16 & 18,0 & 23 & 25,8 & 11 & 12,4 \\
\hline Cali & 67 & 6 & 8,9 & 0 & $\ldots$ & 13 & 19,4 & 6 & 9,0 & 8 & 11,9 \\
\hline Otras & 18 & 1 & 5,5 & 0 & $\ldots$ & 2 & 11,1 & 2 & 11,1 & 1 & 5,6 \\
\hline
\end{tabular}

a TMS = trimetoprima más sulfametoxazol. 
países que notifican casos de SDP todavía no presentan aislamientos con resistencia marcada (24). Las implicaciones clínicas de este hecho son importantes para la elección del antimicrobiano y para determinar las dosis adecuadas porque ambas decisiones dependen de la CIM del aislamiento (7).

La diseminación de la resistencia está determinada, en parte, por el alto consumo de antibióticos en una zona determinada. En este estudio, aproximadamente uno de cada tres aislamientos mostró algún patrón de resistencia; los datos de México indican una proporción aún más alta (25). El hecho de que el alto consumo de antibióticos sin prescripción médica es común en los países de América Latina podría explicar, en alguna medida, la presencia de esos patrones de resistencia. A diferencia de la situación descrita, los países donde se restringe el uso de antibióticos, tales como Alemania e Italia, presentan porcentajes de resistencia bajos (26). Por otra parte, la resistencia a antimicrobianos distintos de la penicilina $(74,5 \%)$ muestra una frecuencia alta similar a la observada en otros países $(3,27)$.

Las cefalosporinas de tercera generación son los antibióticos más utilizados para tratar la meningitis por S. pneumoniae resistente a la penicilina (9). Sin embargo, en los últimos 4 años se han notificado aislamientos con sensibilidad reducida a esos medicamentos; también se han observado fracasos terapéuticos y una evolución clínica desfavorable en pacientes con meningitis cuando la CIM ha sido $>2 \mu \mathrm{g} / \mathrm{mL}$. En este trabajo se encontró una sensibilidad reducida a la ceftriaxona en nueve de los 39 aislamientos con SDP y uno de ellos mostró resistencia alta. Por lo tanto, la circulación de aislamientos con este patrón de resistencia refuerza la necesidad de mantener la vigilancia y establecer así el uso racional de ese antimicrobiano en el medio hospitalario.

El mayor porcentaje de resistencia detectado correspondió al TMS (24,7\%), hallazgo que concuerda con lo notificado en otras partes del mundo. La estrecha asociación entre la resistencia al TMS y a la penicilina también se ha descrito en otros estudios (28).
La resistencia al cloranfenicol encontrada en este trabajo es elevada $(15,1 \%)$, especialmente si se tiene en cuenta que el medicamento es de uso frecuente para tratar casos de meningitis por $S$. pneumoniae en los hospitales colombianos. Países como España y Francia presentan altos porcentajes de resistencia a ese antimicrobiano $(26,29)$, a diferencia de Estados Unidos, donde se restringe el uso de cloranfenicol y el porcentaje de resistencia es bajo (30).

Como en Colombia la resistencia a la eritromicina $(9,6 \%)$ aún no parece ser un problema grave, ese fármaco se podría considerar con mayor frecuencia para tratar las infecciones respiratorias leves y moderadas. Sin embargo, las tasas de resistencia se han elevado considerablemente en los países donde se ha incrementado el uso de nuevos macrólidos $(27,29)$.

La presencia de aislamientos multirresistentes debe alertar al personal de laboratorio y a los clínicos. En esos casos quizá sea conveniente recurrir a agentes como la vancomicina, con las implicaciones que conlleva su utilización. Los datos obtenidos en este estudio sobre la asociación entre la resistencia a la penicilina y la multirresistencia coinciden con lo notificado en otros países $(3,28)$.

Los serotipos asociados con la resistencia a la penicilina son similares en todo el mundo. En general, $60 \%$ de los aislamientos resistentes de niños menores de 2 años corresponden a los tipos capsulares $23 \mathrm{~F}, 14,19 \mathrm{~F}, 6 \mathrm{~A} / 6 \mathrm{~B}$ y 9 (29). En este estudio, $80 \%$ de los aislamientos que presentaron algún tipo de resistencia correspondieron a los tipos capsulares mencionados y al serotipo 5. En los aislamientos resistentes a la penicilina y a otros antimicrobianos predominaron los serotipos $23 \mathrm{~F}$ y 14 , y en el grupo que no era resistente a la penicilina predominó el serotipo 5 . Este hallazgo concuerda con los datos notificados por España (27). Llama la atención la marcada asociación observada en este estudio entre la resistencia al cloranfenicol y el serotipo 5 y sería interesante comprobar si esta asociación se presentó en otros de los países que participaron en el estudio de SIREVA (13).
En este trabajo, el mayor porcentaje de aislamientos con resistencia se encontró en pacientes menores de 2 años, hallazgo similar a lo encontrado en otros países (29). No se encontró ninguna asociación entre los serotipos resistentes y los diferentes diagnósticos clínicos; estos datos difieren de los encontrados en otros estudios que indican que el mayor porcentaje de aislamientos resistentes provenían del líquido cefalorraquídeo $(3,4)$.

Algunos estudios realizados en Estados Unidos y en países europeos, particularmente España y Hungría (23, 26), han revelado diferencias regionales en los patrones y porcentajes de resistencia $(23,26,30)$. Esta situación se presenta con mayor frecuencia en áreas densamente pobladas o donde se prescriben antibióticos en exceso $(26,30)$. Los datos de este estudio confirman esa asociación.

Los factores de riesgo asociados con la presencia de resistencia a los antimicrobianos comprenden no solo los relacionados con la administración inapropiada e indiscriminada de antibióticos, sino también con la edad y la asistencia de los niños a las guarderías infantiles. Por esta razón, las políticas de salud pública deben hacer hincapié en la necesidad de controlar el uso de antimicrobianos, especialmente en la población infantil, donde se presentan los mayores porcentajes de resistencia. También deben subrayar la importancia de controlar las infecciones hospitalarias y las que ocurren en las guarderías infantiles. La experiencia de otros países indica que es posible reducir la resistencia a los antimicrobianos si se crean mecanismos de divulgación que influyan en los hábitos de prescripción y consumo de los antimicrobianos en la comunidad (31).

Agradecimiento. Los autores expresan su agradecimiento a Jorge Jara, epidemiólogo de la Subdirección de Epidemiología del Instituto Nacional de Salud, que colaboró en el análisis estadístico y a José Luis Di Fabio, coordinador del Proyecto SIREVA de la OPS. 
1. Appelbaum PC. Antimicrobial resistance in Streptococcus pneumoniae: an overview. Clin Infect Dis 1992;15(1):77-83.

2. Musher DM. Infections caused by Streptococcus pneumoniae: clinical spectrum, pathogenesis, immunity, and treatment. Clin Infect Dis 1992;14(4):801-807.

3. Linares J, Pallares R, Alonso T, Perez JL, Ayats J, Gudiol F, Viladrich PF, Martin R.Trends in antimicrobial resistance on clinical isolates of Streptococcus pneumoniae in Bellvitge Hospital, Barcelona, Spain (1979-1990). Clin Infect Dis 1992;15(1):99-105.

4. Marton A, Gulyas M, Muñoz R, Tomasz A. Extremely high incidence of antibiotic resistance in clinical isolates of Streptococcus pneumoniae in Hungary. J Infect Dis 1991;163(3): 542-548.

5. Friedland IR, Klugman KP. Antibiotic-resistant pneumococcal disease in South African children. Am J Dis Child 1992;146(8):920-923.

6. Klugman KP. Pneumococcal resistance to antibiotics. Clin Microbiol Rev 1990;3(2):171-196.

7. McCracken GH Jr. Emergence of resistant Streptococcus pneumoniae: a problem in pediatrics. Pediatr Infect Dis J 1995;14(5):424-428.

8. Tan TQ, Schutze GE, Mason EO, Kaplan SL. Antibiotic therapy and acute outcome of meningitis due to Streptococcus pneumoniae considered intermediately susceptible to broadspectrum cephalosporins. Antimicrob Agents Chemother 1994;38(5):918-923.

9. Friedland IR, McCracken GH Jr. Management of infections caused by antibiotic-resistant Streptococcus pneumoniae. N Engl J Med 1994; 331(6):377-382.

10. Hortal M, Palacio R, Camou T, Mogdasy C. Antimicrobial resistance in Streptococcus pneumoniae strains from Uruguay. Pediatr Infect Dis J 1994;13(6):542-543.

11. Sessegolo JF, Levin AS, Levy CE, Asensi M, Facklam RR, Texeira LM. Distribution of serotypes and antimicrobial resistance of Streptococcus pneumoniae strains isolated in Brazil from 1988 to 1992. J Clin Microbiol 1994;32(4): 906-911.

12. Di Fabio JL, Homma A, De Quadros C. Pan American Health Organization epidemiological surveillance network for Streptococcus pneumoniae. Microb Drug Resist 1997;3(2):131-133.
13. Castañeda E, Leal AL, Castillo O, De la Hoz F, Vela MC, Arango M, et al. Distribution of capsular types and antimicrobial susceptibility of invasive isolates of Streptococcus pneumoniae in Colombian children. Pneumococcal Study Group in Colombia. Microb Drug Resist 1997; 3(2):147-152.

14. Sorensen UB. Typing of pneumococci by using pooled antisera. J Clin Microbiol 1993;31 (8):2097-2100.

15. US. National Committee for Clinical Laboratory Standards. Performance standards for antimicrobial disk susceptibility tests. 5th ed. Villanova, Pennsylvania: NCCLS; 1993. (Publication M2-A5).

16. US. National Committee for Clinical Laboratory Standards. Vol X: Methods for dilution antimicrobial susceptibility tests for bacteria that grow aerobically. 2nd ed. Villanova, Pennsylvania: NCCLS; 1990. (Publication M7-A2).

17. Jorgensen JH, Howell AW, Maher LA. Quantitative antimicrobial susceptibility testing of Haemophilus influenzae and Streptococcus pneumoniae by using the E-test. J Clin Microbiol 1991;29(1):109-114.

18. Dean AG, Dean JA, Coulombier D, Brendel KA, Smith DC, Burton AH, Dicker RC, Sullivan K, Fagan RF, Arner TG. Epi Info, Version 6: a word-processing, database, and statistics program for public health on IBM-compatible microcomputers. Atlanta: Centers for Disease Control and Prevention; 1994.

19. Dicker RC. Analyzing and interpreting data. En: Gregg MB. Field epidemiology. NewYork Oxford University Press; 1996.

20. Wust J, Huf E, Kayser FH. Antimicrobial susceptibilities and serotypes on invasive Streptococcus pneumoniae strains in Switzerland. J Clin Microbiol 1995;33(12):3159-3163.

21. Mastro TD, Ghafoor A, Nomani NK, Ishaq Z, Anwar F, Granoff DM at al.. Antimicrobial resistance of pneumococci in children with acute lower respiratory tract infection in Pakistan. Lancet 1991;337(8734):156-159.

22. Leal AL, Castañeda E. Susceptibilidad antimicrobiana de Streptococcus pneumoniae colonizante de nasofaringe en niños colombianos con neumonía. Rev Panam Salud Publica 1997;1(14):266-272.
23. Marton A. Pneumococcal antimicrobial resistance: the problem in Hungary. Clin Infect Dis 1992;15(1):106-111.

24. Reinert RR, Queck A, Kaufhold A, Kresken M, Lutticken R. Antimicrobial resistance and type distribution of Streptococcus pneumoniae isolates causing systemic infections in Germany, 1992-1994. Clin Infect Dis 1995;21(6): 1398-1401.

25. Calderón-Jaimes E, Echaniz-Aviles G, CondeGonzalez C, Rivera-Sanchez R, BarrigaAngulo G, Solórzano-Santos F, et al. Resistencia y serotipificación de 83 cepas de Streptococcus pneumoniae aisladas de niños portadores asintomáticos y enfermos. Bol Med Hosp Infant Mex 1993;50(12):854-860.

26. Baquero F. Pneumococcal resistance to betalactam antibiotics: a global geografic overview. Microb Drug Resist 1995;1(2):115-120.

27. Fenoll A, Martin-Bourgon C, Muñoz R, Vicioso D, Casal J. Serotype distribution and antimicrobial resistance of Streptococcus pneumoniae isolates causing systemic infections in Spain, 1979-1989. Rev Infect Dis 1991;13(1): $56-60$.

28. Geslin P, Buu-Hoi A, Frémaux A, Acar JF. Antimicrobial resistance in Streptococcus pneumoniae: an epidemiological survey in France, 1970-1990. Clin Infect Dis 1992;15(1):95-98.

29. Bédos JP, Chevret S, Chastang C, Geslin P, Régnier B. Epidemiological features and the risk factors for infection by Streptococcus pneumonie strains with diminished susceptibility to penicillin: finding of a French survey. Clin Infect Dis 1996;22(1):63-72.

30. Hofmann J, Cetron MS, Farley MM, Baughman WS, Facklam RR, Elliot JA, Deaver KA, Breiman RF. The prevalence of drug-resistant Streptococcus pneumoniae in Atlanta. N Engl J Med 1995;333(8):481-486.

31. Kristinsson KG. Effect of antimicrobial use and other risk factors on antimicrobial resistance in pneumococci. Microb Drug Resist 1997;3(2):117-23

Manuscrito recibido el 22 de enero de 1998 y aceptado para publicación en versión revisada el 8 de enero de 1999.

\section{ANEXO 1. Grupo Colombiano de Trabajo en Streptococcus pneumoniae}

\section{Centros en Santa Fe de Bogotá:}

Grupo de Microbiología, Instituto Nacional de Salud: CI Agudelo, MC Vela, F de la Hoz, AM Ríos, O Castillo; Hospital Universitario de La Misericordia: M Arango, J Serrato, MR Navarrete, M García, C Lozada; Hospital Infantil Lorencita Villegas de Santos: ME Gama; Hospital de Santa Clara: M Calle, G Aristizábal, A Tovar; Clínica del Niño, ISS: C Paredes, A Arenas; Clínica de Colsubsidio; Hospital de Kennedy; Hospital Simón Bolívar; Hospital San Blas; Clínica CAFAM; Hospital La Granja; Hospital del Tunal; Hospital La Victoria; Laboratorio Central, Secretaría de Salud; Fundación Clínica Shai; Instituto Roosevelt. 


\section{Centros en Medellín:}

Corporación para Investigaciones Biológicas: H Trujillo, GI Mejía, N Correa, J Robledo; Hospital Infantil: ML Valencia, W Parra, N Agudelo, C Zapata; Hospital Pablo Tobón Uribe: S Jaramillo, C Suárez; Hospital General: F Montoya, V García.

\section{Centros en Cali:}

Hospital Universitario del Valle: A Levy, H Porras, A Sánchez, LM Gallardo, N Villamarín. AL Bohorquez, P López; Hospital Infantil Club Noel: D Saa, A Moreno, J Guerrero.

\begin{tabular}{|c|c|}
\hline $\begin{array}{r}\text { Susceptibility to antimicrobial } \\
\text { agents in isolates of invasive } \\
\text { Streptococcus pneumoniae } \\
\text { in Colombia }\end{array}$ & 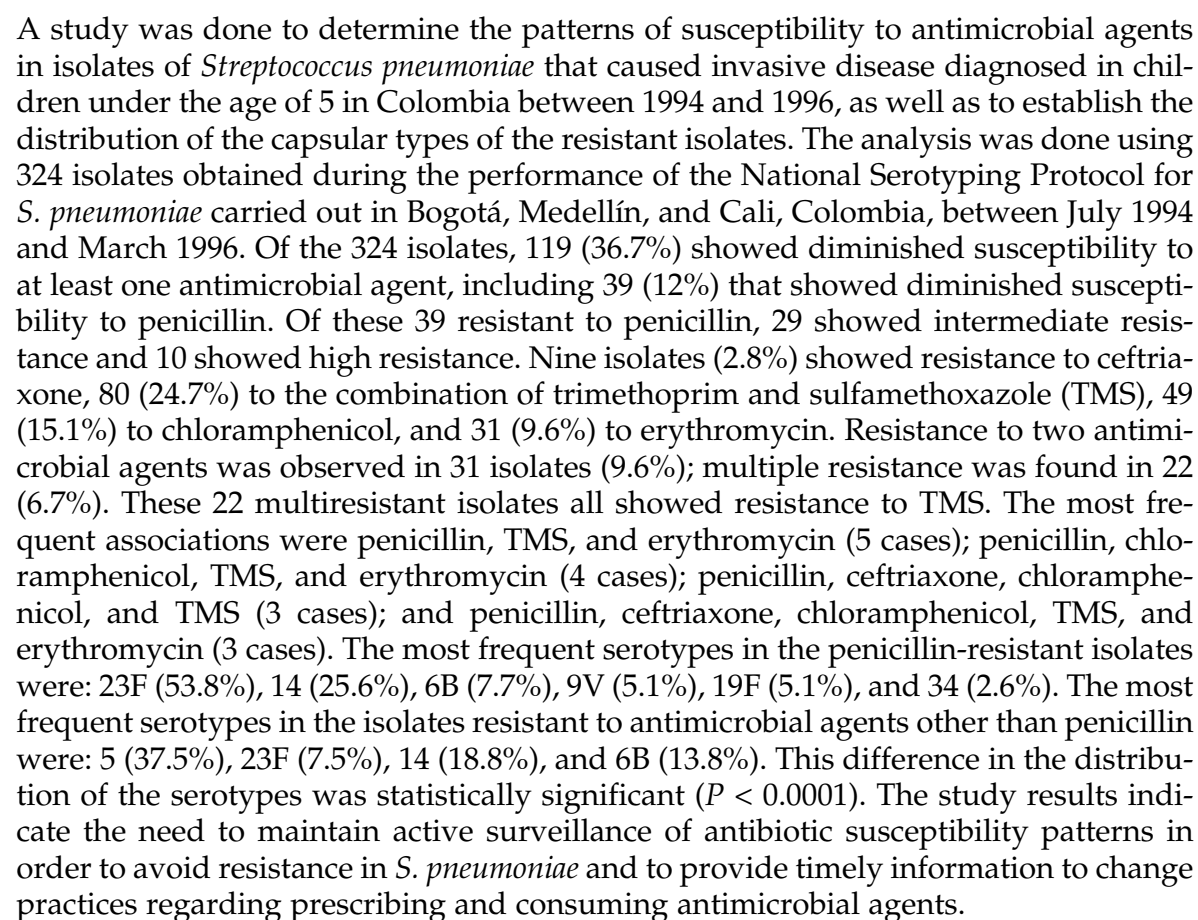 \\
\hline
\end{tabular}

